\title{
Precision Spectroscopy of Kaonic Helium-3 Atoms X-rays at J-PARC
} \author{
Tsukada $^{1}$, A. R. Vidal ${ }^{4}$, E. Widmann ${ }^{3}$, B. Wünschek ${ }^{3}$, T. Yamazaki ${ }^{1,7}$, and J. Zmeskal ${ }^{3}$ \\ 1 RIKEN Nishina Center, RIKEN, Saitama 351-0198, Japan \\ 2 Department of Physics, Seoul National University, Seoul, 151-742, South Korea \\ Stefan Meyer Institut für subatomare Physik, A-1090 Vienna, Austria \\ 4 Laboratori Nazionali di Frascati, INFN, I-00044 Frascati, Italy \\ 5 Research Center for Nuclear Physics (RCNP), Osaka University, Osaka, 567-0047, Japan \\ 6 Department of Physics, Kyoto University Kyoto 606-8502, Japan \\ 7 Department of Physics, The University of Tokyo, Tokyo 113-0033, Japan \\ 8 IPNS, High Energy Accelerator Research Organization (KEK), Ibaraki 305-0801, Japan \\ 9 Department of Physics, Tokyo Institute of Technology, Tokyo, 152-8551, Japan \\ 10 Technische Universiät München, D-85748, Garching, Germany \\ 11 Graduate School of Arts and Sciences, The university of Tokyo, Tokyo, 153-8902, Japan
}

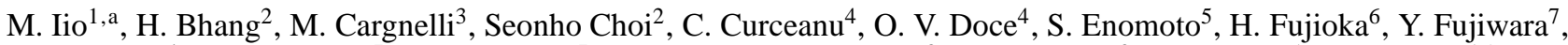
C. Guaraldo ${ }^{4}$, T. Hashimoto ${ }^{7}$, R. S. Hayano ${ }^{7}$, T. Hiraiwa ${ }^{6}$, S. Ishimoto ${ }^{8}$, T. Ishiwatari ${ }^{3}$, K. Itahashi ${ }^{1}$, M. Iwasaki ${ }^{1,9}$, H. $\mathrm{Kou}^{9}$, P. Kienle ${ }^{10}$, J. Marton ${ }^{3}$, Y. Matsuda ${ }^{11}$, H. Noumi ${ }^{5}$, H. Ohnishi ${ }^{1}$, S. Okada ${ }^{4}$, H. Outa ${ }^{1}$, F. Sakuma ${ }^{1}$, M. Sato ${ }^{7}$, M.

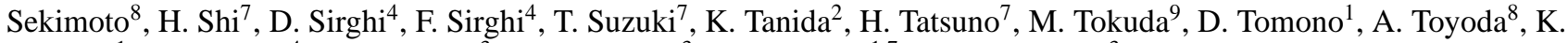

\begin{abstract}
We will measure the Balmer-series x-rays of kaonic- ${ }^{3} \mathrm{He}$ atoms using large-area high-resolution silicon drift $\mathrm{x}$-rays detectors in order to provide the crucial information of $\mathrm{K}^{-}$-nucleus strong interaction at the low energy limit. The strong interaction $2 p$ level shift will be determined with a precision of a few eV. At the present status, the construction of all detectors is in progress. In February, 2009, the first tuning of K1.8BR beamline was performed by the secondary beam generated in J-PARC hadron facility. The data taking will be started soon.
\end{abstract}

\section{Introduction}

The kaonic atom is composed of a nucleus and a negatively charged kaon bound by the Coulomb force. Its energy levels are shifted from their pure electromagnetic values and have a finite absorption width due to the strong interaction between the kaon and the nucleon. The strong interaction last orbital shift and width of the kaonic atoms can be decided by x-ray spectroscopy. This information offers the unique possibility to precisely determine the $\mathrm{K}^{-}$-nucleus strong interaction at vanishing relative energy, and hence many experiments have been done to collect data on various targets, from helium to uranium inclusively [1]. The experimental data of most kaonic atoms except helium and oxygen were in good agreement with the fitting by the optical potential models [2]. The $2 p$-level shift and width of kaonic- ${ }^{4} \mathrm{He}$ atoms have been measured in the past by three experiments. The average of the data determined by the past three kaonic- ${ }^{4} \mathrm{He} \mathrm{x}$-rays measurements [3-5] is to be

$$
\Delta E_{2 p}^{e x p}=-43 \pm 8 \mathrm{eV}, \Gamma_{2 p}^{e x p}=55 \pm 34 \mathrm{eV}
$$

\footnotetext{
a e-mail: iio@riken.jp
}

while a majority of theoretical calculations $[2,6,7]$ estimates to be

$$
\Delta E_{2 p}^{o p t}=-0.2 \mathrm{eV}, \Gamma_{2 p}^{o p t}=2 \mathrm{eV}
$$

As to this discrepancy, there has been a long debate, the so-called "kaonic helium puzzle".

The kaonic helium puzzle has recently attracted a renewed interest in connection with the Akaishi-Yamazaki prediction of "deeply-bound kaonic nuclei system" [8,9]. $\Lambda(1405)$ is treated in a negatively charged kaon and proton $\left(K^{-} p\right)$ bound state by coupled channel approach, which predict unconventionally deep $\mathrm{K}^{-}$-nucleus potentials. The framework allows the large shift $\left(\Delta \mathrm{E}_{2 p} \sim 10 \mathrm{eV}\right)$ of the kaonic- ${ }^{4} \mathrm{He}$ and kaonic- ${ }^{3} \mathrm{He}$ atoms [10]. Furthermore, they expected a difference in the value of shift between kaonic${ }^{4} \mathrm{He}$ and kaonic- ${ }^{3} \mathrm{He}$ by an anomalous $2 p$ repulsive shift for $\mathrm{K}^{-}{ }^{4} \mathrm{He}$ and an anomalous $2 p$ attractive shift for $\mathrm{K}^{-}$${ }^{3} \mathrm{He}$. Therefore precise measurement of the $2 p$ shifts of both kaonic- ${ }^{4} \mathrm{He}$ and kaonic- ${ }^{3} \mathrm{He}$ has been awaited.

This situation motivated to perform measurement of kaonic- ${ }^{4} \mathrm{He}$ atoms Balmer-series X-rays at the $\mathrm{K} 5$ beamline of the KEK 12-GeV proton synchrotron (KEK-PS E570). As a result, the value of the $2 p$ level shift of kaonic- ${ }^{4} \mathrm{He}$ 
was determined to be

$$
\Delta E_{2 p}^{E 570}=+2 \pm 2(\text { stat }) \pm 2(\text { syst }) \mathrm{eV} .
$$

The careful and precise result excludes the earlier claim of the large shift of about $-40 \mathrm{eV}$ [11]. However, no data exist yet on the kaonic- ${ }^{3} \mathrm{He} \mathrm{x}$-rays. Therefore, we perform a precision spectroscopy of kaonic- ${ }^{3} \mathrm{He} 3 d \rightarrow 2 p$ x-rays at Japan Proton Accelerator Research Complex (J-PARC) [12].

\section{Experimental method}

E17 is one of the Day-1 experiments performed in J-PARC hadron facility. The goal of E17 is to measure the $2 p$ level shift with a precision better than $\sim 2 \mathrm{eV}$ using similar technique to that of KEK-PS E570 [11]. Figure 1 shows a schematic view of the E17 experimental setup around the target. The kaonic- ${ }^{3} \mathrm{He}$ atom is generated by negative kaons stopped inside the ${ }^{3} \mathrm{He}$ target (cylindrical shape 68 $\mathrm{mm}$ in diameter and $138 \mathrm{~mm}$ long at a density of 0.081 $\mathrm{g} / \mathrm{cm}^{3}$ ). Incident kaons with momentum $0.75 \mathrm{GeV} / \mathrm{c}$ are degraded in carbon degraders, counted with beamline counters, tracked by a high-rate beamline drift chamber. The energy loss before stopping is measured in a set of scintillation counters. X-rays emitted from the kaonic- ${ }^{3} \mathrm{He}$ atoms are detected by eight $\mathrm{X}$-ray detectors which view the target from round through the $0.3 \mathrm{~mm}$-thick berylium cylinder of the target cell. Secondary charged particles produced in the kaon absorption process is detected by cylindrical detector system for reducing background events. The three main special techniques are written in the following subsections each.

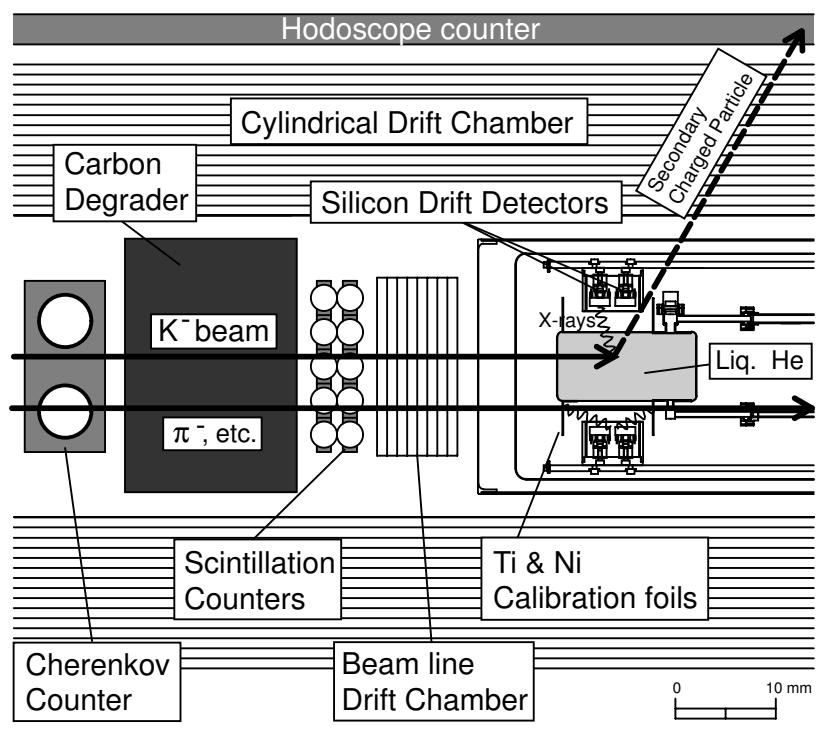

Fig. 1. A schematic side view of the E17 setup around the cylindrical target with the x-ray detection system. Two SDDs are mounted in one housing, and four housings are put to surround the target cell. High-purity titanium and nickel foils are put in the shape of a ring on the edge of the cell.

\subsection{High resolution x-ray detector}

Instead of a conventional $\mathrm{Si}(\mathrm{Li}) \mathrm{X}$-ray detector, Silicon Drift Detectors (SDDs) produced by KETEK [13] are adopted for energy resolution improvement of x-ray detection. A photograph of the SDD shown in figure 2. Each SDD has an effective area of $100 \mathrm{~mm}^{2}$ and a $450 \mu \mathrm{m}$-thick active layer. The temperature of the SDDs is kept at $\sim 150 \mathrm{~K}$ during the experimental period by a connection to the thermalradiation shield for the liquid ${ }^{3} \mathrm{He}$ target cooled by liquid nitrogen. Typical energy resolution is $\sim 150 \mathrm{eV}$ (FWHM) at $6.4 \mathrm{keV}$, which is about twice as good as that of the $\mathrm{Si}(\mathrm{Li})$ $\mathrm{X}$-ray detectors used in the past three experiments [3-5]. The time resolution is about $160 \mathrm{nsec}$ (rms). In the SDD, the electrons produced by an X-ray hit drift radially toward the central anode where they are collected. The small anode size (and hence small capacitance) is essential to realize the good energy resolution despite the large effective area. The small anode area also makes it possible to reduce the active layer thickness, while the capacitance is still kept small. The thin active layer helps to reduce continuum background caused by the Compton scattering occurring inside the detector.

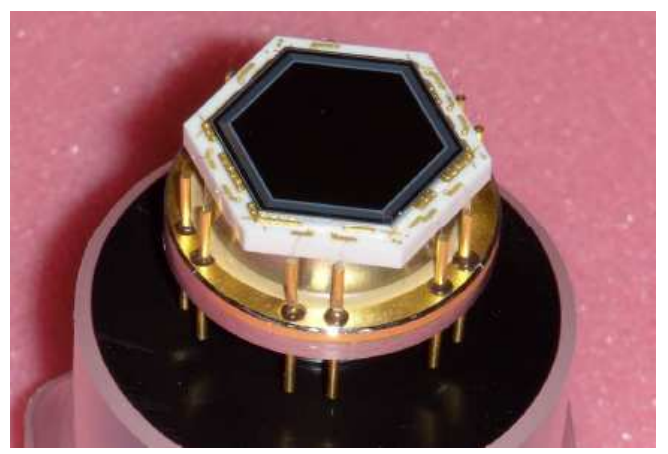

Fig. 2. A photograph of the hexagonal Silicon Drift Detector (SDD) produced by KETEK $\left(100 \mathrm{~mm}^{2}\right)$.

\subsection{Cuts for reducing background events}

The $\mathrm{x}$-ray events coming from the kaonic- ${ }^{3} \mathrm{He}$ transitions are selected by the reaction vertices reconstructed from an incident kaon track and a second charged particle track, which is called the "fiducial volume cut". Moreover, inflight kaon decay and reaction events are rejected by applying a correlation cut between the z-coordinate of the reaction vertex and the energy loss in scintillation counters. As a result of KEK-E570, a good signal-to-noise ratio of $\sim 4$ was achieved, which is about 5 times better than that of the past experiments.

\subsection{In-beam energy calibration}

The transition energies are calibrated by using characteristic $\mathrm{x}$-rays induced by beam particles on high-purity titanium and nickel foils placed around the target cell. The 
$19^{\text {th }}$ International IUPAP Conference on Few-Body Problems in Physics

energy of the kaonic- ${ }^{3} \mathrm{He} 3 d \rightarrow 2 p$ x-ray, $\sim 6.2 \mathrm{keV}$, lies between these characteristic x-ray energies of $4.5 \mathrm{keV}(\mathrm{Ti})$ and $7.5 \mathrm{keV}(\mathrm{Ni})$. We accumulate SDD self-triggered events together with the stopped- $\mathrm{K}^{-}$triggered events, which provide high-accuracy in-situ calibration spectra. To avoid detecting the background characteristic x-rays from other than the titanium and nickel, high-purity aluminum foils are placed on all objects in the view of the SDDs.

\section{Detectors construction}

E15 $[14,15]$ and E17 experiments are perform precision studies of the interaction between kaons and nucleons/nuclei at K1.8BR beamline in J-PARC hadron facility. Addressing items as kaonic atoms and deeply-bound kaonic nuclear states, these experiments are closely related and there is a large overlap of the collaboration members.

Both experiments use the same beamline, a liquid ${ }^{3} \mathrm{He}$ target and a cylindrical detector system. The setup of E15/$\mathrm{E} 17$ in K1.8BR experimental area shown in figure 3. The E17 detectors consist of beamline spectrometer, cylindrical detector system, liquid ${ }^{3} \mathrm{He}$ target and silicon drift $\mathrm{X}$-rays detectors. The construction status of each detectors is written in follows.

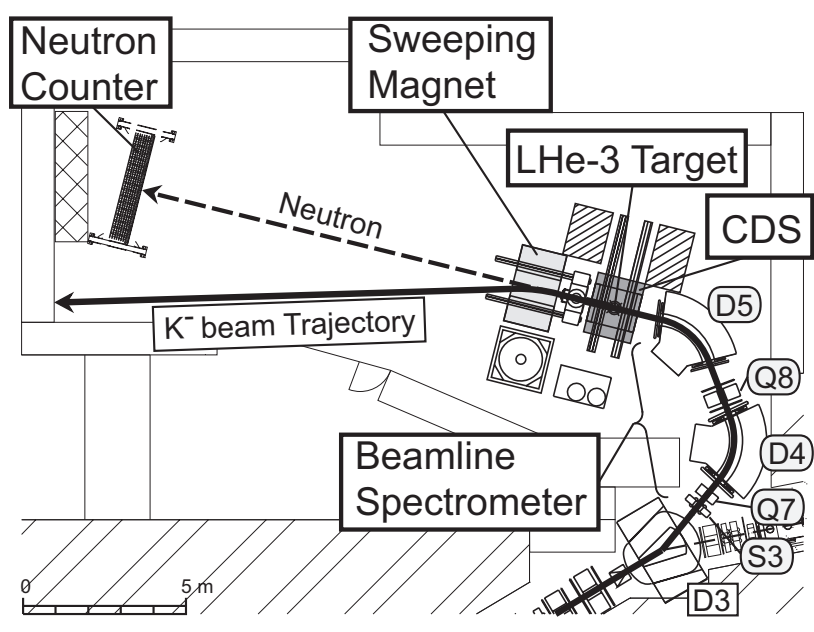

Fig. 3. The layout of K1.8BR experimental area. The magnet constitution of the beamline spectrometer section consists of a sextupole (S3), two quadrupoles (Q7, Q8)and two dipoles (D4, D5). In E17 for stopped- $\mathrm{K}^{-}$reaction, a sweeping magnet placed downstream of the target is not used.

\subsection{Beamline Spectrometer}

K1.8BR beamline was designed to perform the experiment using the secondary particles $\left(\pi^{ \pm}, K^{ \pm}, \bar{p}\right.$, etc). The particles produced in a production target are momentum-analyzed and collected by magnetic optical elements and electrostatic separator. Table 1 lists the expected performance of K1.8BR beamline. The downstream section of a splitter
Table 1. A expected performance of K1.8BR beamline

\begin{tabular}{lrr}
\hline & \multicolumn{2}{c}{ DAY-I $(30 \mathrm{GeV}, 9 \mu \mathrm{A})$} \\
\hline Maximum momentum & {$[\mathrm{GeV} / \mathrm{c}]$} & 1.10 \\
Production target (Ni) thickness & {$[\mathrm{mm}]$} & 54 \\
Beamline length & {$[\mathrm{m}]$} & 31.412 \\
Acceptance & {$[\mathrm{msr} \% \%]$} & 2.5 \\
$\mathrm{~K}^{-}$intensity for $1.10 \mathrm{GeV} / \mathrm{c}$ & {$[/ \mathrm{pulse}]$} & $770 \times 10^{3}$ \\
$\mathrm{~K}^{-}$intensity for $0.75 \mathrm{GeV} / \mathrm{c}$ & {$[/ \mathrm{pulse}]$} & $66 \times 10^{3}$ \\
\hline
\end{tabular}

magnet (D3) behaves as a beamline spectrometer which consists of beamline magnets, hodoscopes, drift chambers, timing counters and Cherenkov counters. The construction of all detectors was completed and installed between the magnets as follows.

- In consideration of a high rate of particle hits, beam hodoscope (BHD) segmented 20 of 20 mm-width, 170 $\mathrm{mm}$-height, $5 \mathrm{~mm}$-thick was installed between D3 and S3.

- Scintillation counters stack segmented 8 of $32 \mathrm{~mm}$ width, $260 \mathrm{~mm}$-height, $8 \mathrm{~mm}$-thick and 2 set of drift chamber $8 \mathrm{~mm}$-spacing 32 wires each (xx'yy') were installed between Q8 and D5.

- 2 set of drift chamber $5 \mathrm{~mm}$-spacing 32 wires each (xx'yy') and timing counters segmented 5 of $32 \mathrm{~mm}$ width, $165 \mathrm{~mm}$-height, $10 \mathrm{~mm}$-thick (T0) were installed downstream of D5. During beam tuning, to identify all particles $(p, K, \pi(\mu), \mathrm{e})$ of 0.75 and $1.0 \mathrm{GeV} / \mathrm{c}$ momenta at a hardware stage, we installed the Cherenkov detectors of aerogel, water and gas (CFC) temporarily.

In February, 2009, the first tuning of the beamline was performed by the secondary beam generated in J-PARC hadron facility. The tuning plan has a large number of items due to different parameter settings each experiment. The items are shown in table 2 . We started the tuning from setting of $1.1 \mathrm{GeV} / \mathrm{c}$ unseparated positive beam first. Acquisition of beam profiles, analysis of time structures and separating of secondary particles were performed sequentially. As a conclusive result of 2009 run, positively charged kaons were identified by a time of flight measurement from beam hodoscopes (BHD) to timing counters (T0). The distance of BHD and T0 is $7.7 \mathrm{~m}$ and the calculated value of TOF difference between kaon and pion at $1.1 \mathrm{GeV} / \mathrm{c}$ is $2.3 \mathrm{nsec}$. The TOF spectrum after slewing correction and offset tune is shown in figure 4 . The peak structure was obtained from the peak of the light particle $\left(e^{+}, \mu^{+}, \pi^{+}\right)$for $2.4 \mathrm{nsec}$. Therefore, we succeeded in the separation of kaon and other particles. In the next beam time from the autumn of 2009, tuning of $0.75 \mathrm{GeV} / \mathrm{c}$ will be started for E17 experiment.

\subsection{Cylindrical detector system}

One of the main detectors for experimental search for deeply-bound kaoinc nuclear state $K^{-} p p$ (J-PARC E15) [14] is a Cylindrical Detector System (CDS). A schematic view of the CDS is shown in figure 5. The CDS is developed to detect the decay particles from the expected decay of 
Table 2. The items of K1.8BR tuning plan

\begin{tabular}{l|r|r}
\hline Condition/Item & E15 (Nuclei) & E17 (Atoms) \\
\hline Beam particles & $\mathrm{K}^{-}$ & $\mathrm{K}^{-}$ \\
Momentum $(\mathrm{GeV} / \mathrm{c})$ & 1.00 & 0.75 \\
Reaction & in-flight & stopped \\
Maximization of kaons & necessity & necessity \\
Optimization of $\mathrm{K} / \pi$ ratio & necessity & necessity \\
Momentum measurement & necessity & necessity \\
Range measurement & unnecessity & necessity \\
\hline
\end{tabular}

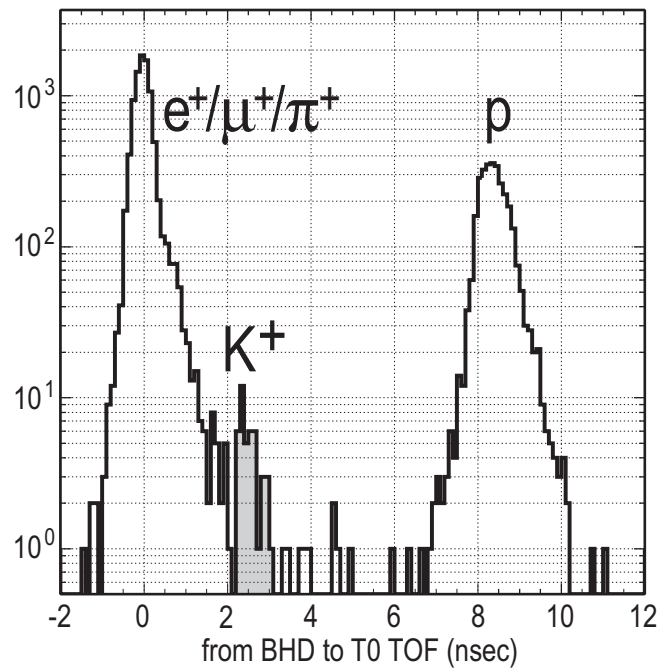

Fig. 4. A time of flight (TOF) spectrum from beam hodoscopes (BHD) to timing counters (T0) for $\mathrm{K}^{+}$identification. The distance of BHD and $\mathrm{T} 0$ is $7.7 \mathrm{~m}$.

kaonic nucleus $K^{-} p p \rightarrow \Lambda p \rightarrow \pi^{-} p p$. Tracking is preformed with a Cylindrical Drift Chamber (CDC) which operates in a magnetic field of $0.5 \mathrm{~T}$ provided by the solenoid magnet. A Cylindrical Detector Hodoscope (CDH), which surrounds the $\mathrm{CDC}$, is used for charged particle trigger and particle identification. The expected spectrometer performance for the $K^{-} p p$ measurement is $\sim 20 \mathrm{MeV} / \mathrm{c}^{2}(\sigma)$ for the invariant-mass resolution where we assume the $K^{-} p p$ binding energy to be $100 \mathrm{MeV} / \mathrm{c}^{2}$. The details for E15 and the present status of commissioning are written in Ref. [15].

In E17, the system is used for fiducial volume cut to reduce the background $\mathrm{x}$-ray events without magnetic field. Secondary charged particles produced in the kaon absorption process are triggered by the $\mathrm{CDH}$, the tracks are measured by the CDC. The angle of the CDS from the center is about $7.4 \mathrm{sr}$.

\subsubsection{Cylindrical detector hodoscope}

The Cylindrical Detector Hodoscope system using plastic scintillation counters is used for the secondary charged particle trigger. The $\mathrm{CDH}$ system is located at a radius of 544 $\mathrm{mm}$ from the target system covering a polar angle range from 54 to 126 degrees. The CDH system consists of 36 modules, and these modules are individually mounted on

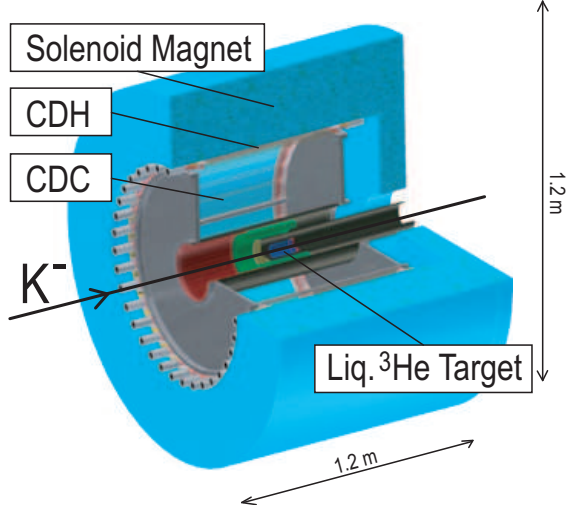

Fig. 5. A schematic view of the cylindrical detector system.

the inner wall of the solenoid magnet as shown in figure. 6 . The dimensions of the scintillators are $790 \mathrm{~mm}$-length, $99 \mathrm{~mm}$-width and $30 \mathrm{~mm}$-thick. Fine-mesh photomultipliers, with a 1.5 inch-diameter and 19 dynode-stages, are selected. Though it is not supplied the magnetic field in E17, the operation of the $\mathrm{CDH}$ are assumed under the magnetic field of $0.5 \mathrm{~T}$, with a typical gain of $3 \times 10^{6}$. The construction of $\mathrm{CDH}$ has been completed.

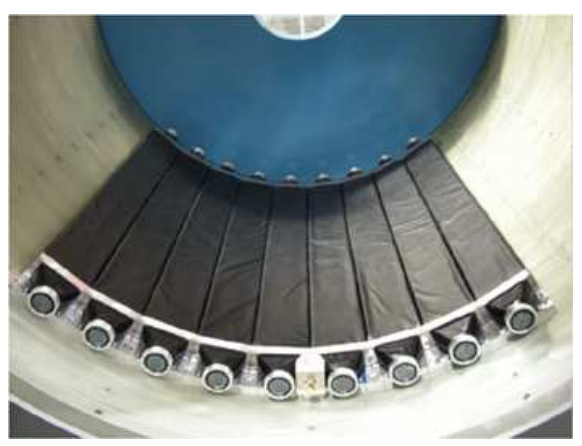

Fig. 6. A photograph of the installation of the cylindrical detector hodoscope.

\subsubsection{Cylindrical drift chamber}

The Cylindrical Drift Chamber (CDC) occupies most of the region inside the solenoid magnet starting at a radial distance of $150 \mathrm{~mm}$ from the beam axis up to $530 \mathrm{~mm}$. The total length of the CDC is $950 \mathrm{~mm}$. The CDC consists of 15 layers of small hexagonal cells with typical drift length of $9 \mathrm{~mm}$, which are grouped into 7 super layers (A1, $\mathrm{U} 1, \mathrm{~V} 1, \mathrm{~A} 2, \mathrm{U} 2, \mathrm{~V} 2, \mathrm{~A} 3)$. Longitudinal position information is obtained from 8 stereo layers which have small angle with respect to the $\mathrm{z}$-axis. The number of the readout channels is 1816 and the total number of wires in the CDC is 8064 . The $\operatorname{argon}(50 \%)$ and ethane $(50 \%)$ mixed gas is used at $1 \mathrm{~atm}$. A photograph of the CDC is shown in figure 7. Position resolutions of the $\mathrm{CDC}$ at the target position are expected with $0.2 \mathrm{~mm}(\sigma)$ in plane perpendicular to the $\mathrm{z}$ axis and $6 \mathrm{~mm}(\sigma)$ in the $\mathrm{z}$-direction by the straight track 
without magnetic field. The $\mathrm{CDC}$ has been constructed and the commissioning is going on at J-PARC.

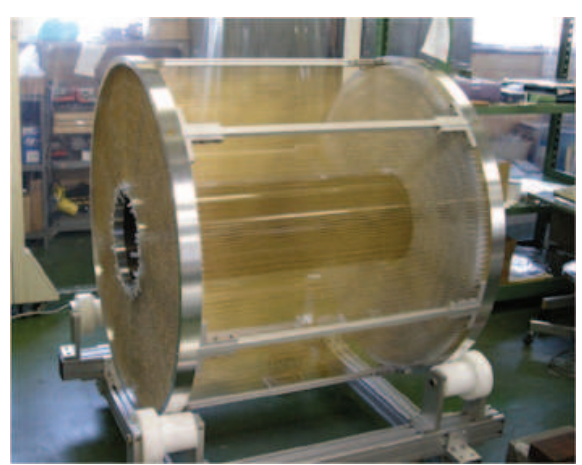

Fig. 7. A photograph of the cylindrical drift chamber.

\subsection{Liquid ${ }^{3} \mathrm{He}$ target}

A liquid ${ }^{3} \mathrm{He}$ target has been developed for E15 experiment as the joint project between KEK and RIKEN. Figure 8 shows the design of the liquid ${ }^{3} \mathrm{He}$ target. The target was designed by L-form to place a target cell in the center of the Cylindrical detector system. Basic design for the target system is based on the techniques developed for the superfluid ${ }^{4} \mathrm{He}$ target used by KEK-PS E471, E549 and E570 [17]. Liquid ${ }^{4} \mathrm{He}$ is supplied to the ${ }^{4} \mathrm{He}$-separator ( 4 K) inside the target system from a 1000 liter dewar placed outside by a transfer tube. From the separator, only liquid ${ }^{4} \mathrm{He}$ drop into the ${ }^{4} \mathrm{He}$-evaporator which is placed under the separator. The inside of evaporator is decompressed down to $\sim 1$ Torr, the temperature is kept $\sim 1.3 \mathrm{~K}$. The ${ }^{3} \mathrm{He}$ gas is cooled down and liquefied in the ${ }^{3} \mathrm{He}$-heat exchanger which is placed under the ${ }^{4} \mathrm{He}$-evaporator. The target cell almost $1 \mathrm{~m}$ away from cryostat is filled with liquid ${ }^{3}$ liquefied in the ${ }^{3} \mathrm{He}$-heat exchanger.

The cooling tests were performed at K5 experimental area in KEK-PS north counter hall. The setup of cooling system was the almost same as production run setting for E15, and 250 liters ${ }^{3} \mathrm{He}$ gas was used as the target material on the test. The results of the test is summarized in table 3 . The target cell was cooled down and kept at $\sim 1.3 \mathrm{~K}$. Since the pressure in the evaporator and cell is also measured at the same time, we were able to study the correlation of the pressure and temperature. The result is shown in figure 9. Where black and white dots are correspond to the cell and evaporator. Although there are small shift by the error of the measuring device, the both measurements are good agreement with vapor pressure curve. It is clear that liquid ${ }^{3} \mathrm{He}$ exists in the target cell. In addition, the heat load to the $1 \mathrm{~K}$ parts is $0.19 \mathrm{~W}$, high-performance cooling system for E15 liquid ${ }^{3} \mathrm{He}$ target was completed.

\subsubsection{Upgrading for E17 experiment}

In E17 experiment, the SDDs are installed in the target system in consideration of attenuation of kaonic- ${ }^{3} \mathrm{He} \mathrm{x}$-rays.

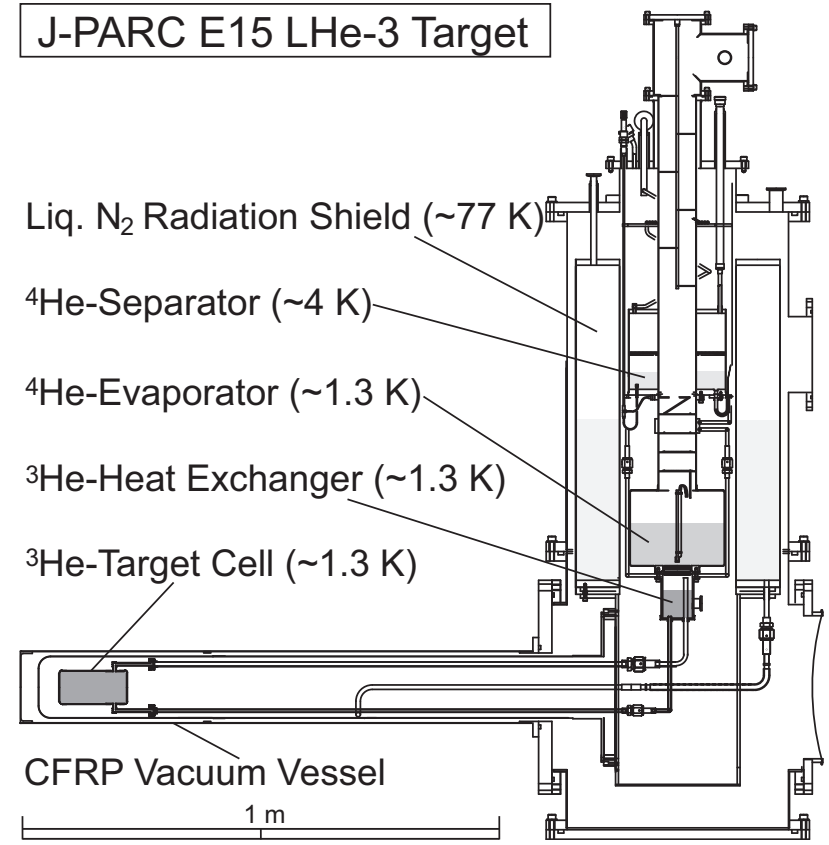

Fig. 8. A schematic side view of the E15 liquid ${ }^{3} \mathrm{He}$ target.

Table 3. The results of the cooling test of the E15 Liquid ${ }^{3} \mathrm{He}$ target

\begin{tabular}{lr|r}
\hline \multicolumn{2}{c|}{ Condition/result } & \\
\hline Amount of target material & ${ }^{3} \mathrm{He}$ gas [liter] & $250 / 450$ \\
Temp. in the evaporator & $\left({ }^{4} \mathrm{He}\right)[\mathrm{K}]$ & 1.3 \\
Temp. in the heat exchanger & $\left({ }^{3} \mathrm{He}\right)[\mathrm{K}]$ & 1.3 \\
Temp. in the target cell & $\left({ }^{3} \mathrm{He}\right)[\mathrm{K}]$ & 1.3 \\
Press. in the target cell & {$[\mathrm{mbar}]$} & 33 \\
Liq. ${ }^{4} \mathrm{He}$ consumption & {$[$ liter/day] } & 45 \\
Heat load to the 1K parts & {$[\mathrm{W}]$} & 0.19 \\
\hline
\end{tabular}

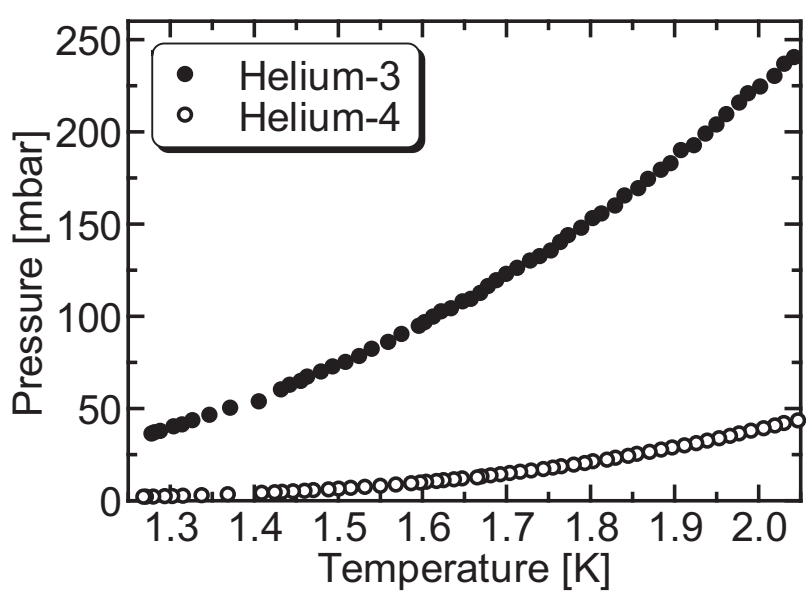

Fig. 9. The measurements of the vapor pressure curve by the correlation of the pressure and temperature in the cell and evaporator.

Though the pre-amps for SDD were put in the KEK-E570 experiment outside of the target, they are also installed in the target by spatial limitation. Power to work the eight sets of SDD and pre-amps is $\sim 10 \mathrm{~W}$, the temperature condi- 
tions are SDD in $\sim 150 \mathrm{~K}$, and the pre-amp in almost roomtemperature. Therefore, the huge heat load to the cryogenic system is serious problem. To solve the problem, we adopt a method that SDDs and pre-amps are put inside a box which is kept at $\sim 80 \mathrm{~K}$ by liquid nitrogen. The upgrading for E17 of taget system is shown in figure 10. If $1 \mathrm{~K}$ part such as the target cell, heat exchanger and evaporator has no directly-contact with SDDs and pre-amps thermally, the heat load caused by the radiation is dominant. In hiding the $1 \mathrm{~K}$ part from hot part, the radiation is negligible small. We performed the test using the dummy heater in substitution for the pre-amp due to the confirmation of our method. As a result, we confirmed that the target cell was filled with liquid ${ }^{4} \mathrm{He}$, and it was kept at $1.5 \mathrm{~K}$ under the temperature of dummy pre-amp in $220 \mathrm{~K}$ loaded of $15 \mathrm{~W}$. About this heat load test, the details are written in Ref. [16].

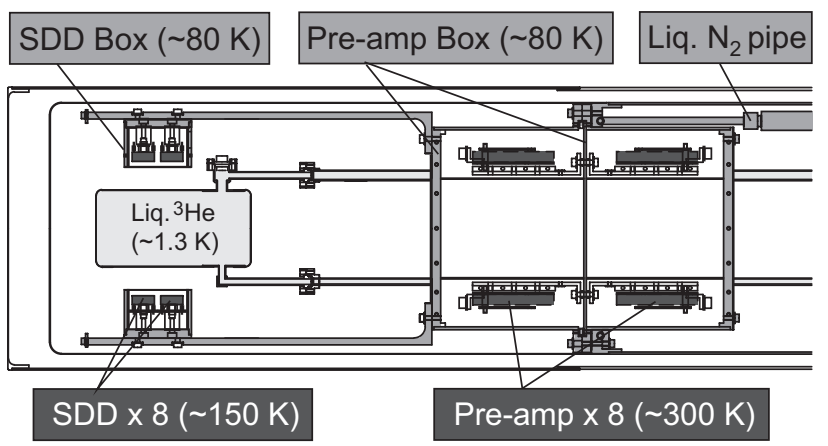

Fig. 10. The upgrading for reducing the heat load to the cryogenic system by the instration of the $\mathrm{x}$-ray detection device.

\subsection{Silicon drift x-rays detectors}

New eight sets of SDD and pre-amp produced by KETEK were adopted for energy resolution improvement in E17 [13]. The characteristics of the new SDD set were studied with a test bench shown in figure 11 before installation to the target system. The parameters search for optimization of the energy resolution, temperature dependence of SDD and pre-amp each [16], x-ray hit position and incident angle dependence of effective area are studied at K5 experimental area in KEK, and the results are fed back to the development of the target. A typical energy spectrum of the new SDD using ${ }^{55} \mathrm{Fe}$ source is shown in figure 12. The temperature of SDD and pre-amp is $140 \mathrm{~K}$ and 250 $\mathrm{K}$ each. The distinguishing double peaks of Mn $K_{\alpha}$ and $K_{\beta}$ are clearly seen in the spectrum. The energy resolution fitted with gauss and tail function for $K_{\alpha} / K_{\beta}$ is $\sim 150 \mathrm{eV}$ (FWHM), the resolution improved than prototype SDD in $190 \mathrm{eV}$ (FWHM) which used in KEK-E570. Currently, test of the SDD set which installed in the main target system is performed at KEK. Then, the liquid ${ }^{3} \mathrm{He}$ target and SDD system are transferred to J-PARC.

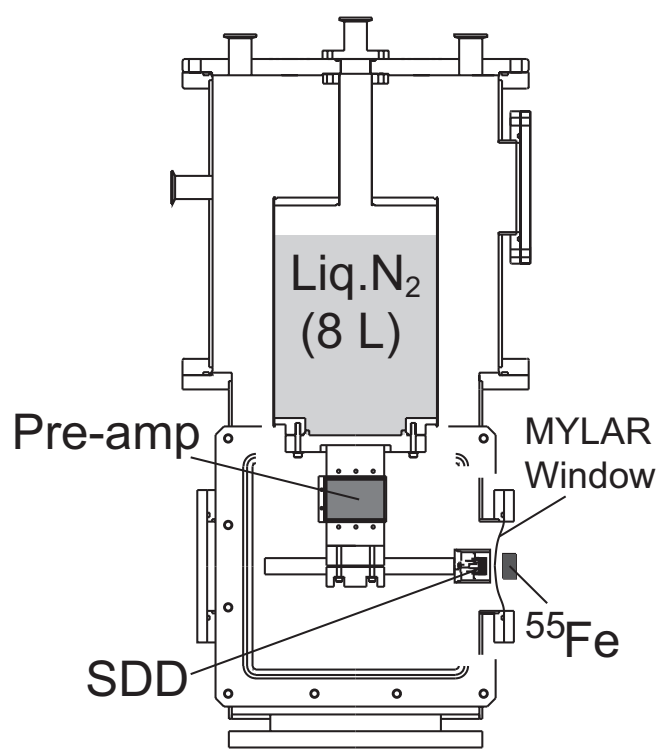

Fig. 11. A schematic side view of the test bench for SDD studies.

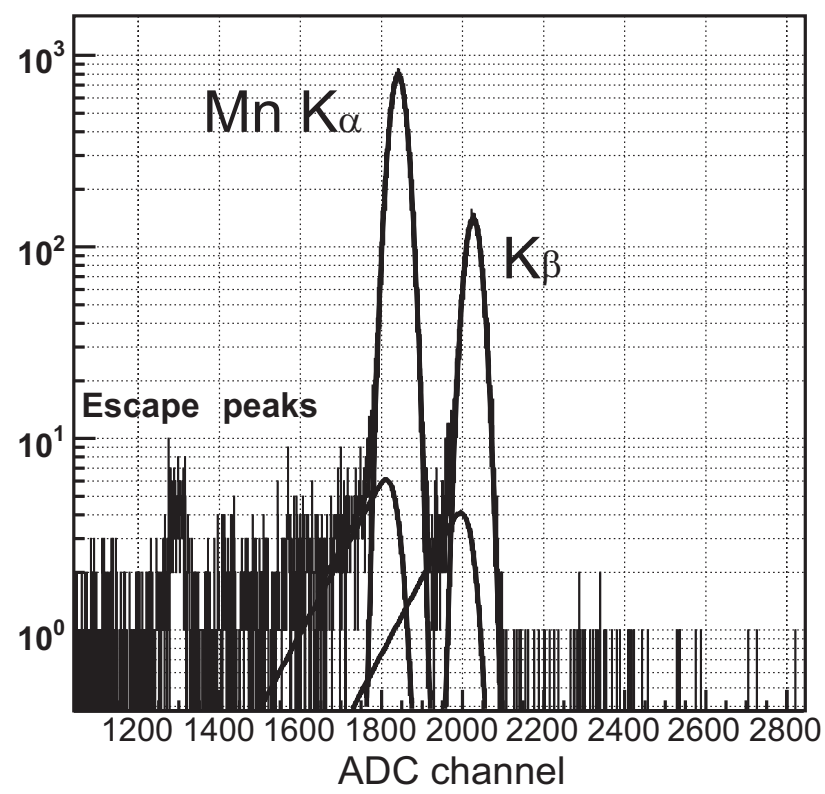

Fig. 12. A typical new SDD energy spectrum for ${ }^{55} \mathrm{Fe}$ source calibration. The resolution is $\sim 150 \mathrm{eV}$ (FWHM) at $6.4 \mathrm{keV}$.

\section{Summary and Outlook}

The Balmer-series $\mathrm{x}$-rays of kaonic- ${ }^{3} \mathrm{He}$ atoms will be measured at K1.8BR beamline in J-PARC hadron facility. The strong interaction shift of $2 p$ level will be determined with precision of a few $\mathrm{eV}$ using similar technique to that of KEK-E570 experiment. Silicon drift detectors are adopted for energy resolution improvement of $\mathrm{x}$-ray detection. The energy resolution of SDD was confirmed with $\sim 150 \mathrm{eV}$ (FWHM) at $6.4 \mathrm{keV}$ using ${ }^{55} \mathrm{Fe}$ source. The $\mathrm{x}$-ray events coming from the kaonic- ${ }^{3} \mathrm{He}$ transitions are selected by the reaction vertices reconstructed from an incident kaon track and a second charged particle track which come from the liquid ${ }^{3} \mathrm{He}$ target for reducing background events. The 
$19^{\text {th }}$ International IUPAP Conference on Few-Body Problems in Physics

transition energies are calibrated by using characteristic $\mathrm{x}$ rays induced by beam particles on high-purity titanium and nickel foils placed around the target cell. The E17 detectors consist of beamline spectrometer, cylindrical detector system, liquid ${ }^{3} \mathrm{He}$ target and silicon drift $\mathrm{x}$-rays detectors. The detector construction is in progress. The construction of all detectors for beamline spectrometer was completed and installed between the beamline elements. The construction of cylindrical detector system has been completed. High performance cooling system for liquid ${ }^{3} \mathrm{He}$ target was completed. The target is upgraded for instllation x-ray detactor system to inside. In February, 2009, the first tuning of K1.8BR beamline was performed by the secondary beam generated in hadron facility. As a conclusive result of 2009 run, positively charged kaons were identified by TOF measurement. In the next beam time from the autumn, 2009, tuning of $0.75 \mathrm{GeV} / \mathrm{c}$ will be started for E17 experiment. The data taking for E17 experimet at J-PARC will be started soon.

\section{References}

1. C.J. Batty, E. Friedman, A. Gal, Phys. Reports. 287, (1997) 385.

2. S. Hirenzaki, Y. Okumura, H. Toki, E. Oset, and A. Ramos, Phys. Rev. C61, (2000) 055205.

3. C.E. Wiegand, R. Pehl, Phys. Rev. Lett. 27 (1971) 1410.

4. C.J. Batty, et al., Nucl. Phys. A 326 (1979) 445.

5. S. Baird, et al., Nucl. Phys. A 392 (1983) 297.

6. C.J. Batty, Nucl. Phys. A 508 (1990) 89c.

7. E. Friedman, private communication

8. Y. Akaishi and T. Yamazaki, Phys. Rev. C 65, (2002) 044005.

9. T. Yamazaki and Y. Akaishi, Phys. Lett. B 535, (2002) 70.

10. Y. Akaishi, Proceedings for International Conference on Exotic Atoms and Related Topics (EXA05), Austrian Academy of Sciences Press, Vienna, 2005, p. 45. (http://dx.doi.org/10.1553/exa05s45)

11. S. Okada, et al., Phys. Lett. B 653,1 (2007) 387-39.

12. R.S. Hayano, et al., Proposal of J-PARC 50-GeV PS "Precision spectroscopy of Kaonic Helium $33 d \rightarrow 2 p$ $X$-rays”, (2006).

13. http://www.ketek.net.

14. M. Iwasaki, et al., Proposal of J-PARC 50-GeV PS “A search for deeply-bound kaonic nuclear states by inflight ${ }^{3} \mathrm{He}\left(\mathrm{K}^{-}, n\right)$ reaction", (2006).

15. K. Tsukada, et al., for J-PARC E15 collaboration, contribution to this conference.

16. Y. Fujiwara, et al., for J-PARC E17 collaboration, contribution to this conference.

17. M. Sato, et al., Nucl. Instr. and Meth. A 606 (2009) 233-237. 